NBER WORKING PAPER SERIES

\title{
OPPORTUNITIES FOR IMPROVING \\ THE DRUG DEVELOPMENT PROCESS: \\ RESULTS FROM A SURVEY OF INDUSTRY AND THE FDA
}

\author{
Ernst R. Berndt \\ Adrian H. B. Gottschalk \\ Matthew W. Strobeck \\ Working Paper 11425 \\ http://www.nber.org/papers/w11425
}

\author{
NATIONAL BUREAU OF ECONOMIC RESEARCH \\ 1050 Massachusetts Avenue \\ Cambridge, MA 02138 \\ June 2005
}

Mr. Berndt is the Louis B. Seley Professor of Applied Economics at the MIT Sloan School of Management and Director of the National Bureau of Economic Research Program on Technological Progress and Productivity Measurement. The research reported here fulfilled in part the 2004 S.M. thesis requirements for Mr. Gottschalk and Mr. Strobeck at the Harvard-MIT Division of Health Sciences and Technology. Funding support from the Harvard-MIT Division of Health Sciences and Technology is gratefully acknowledged, as is sabbatical leave support to Mr. Berndt from MIT. We have benefited from the comments of Joseph Bonventre, John Grana, Rebecca Henderson, Genia Long, and Scott Stern. Particular thanks are due Mark McClellan, M.D., Ph.D., and Thomas Philipson, Ph.D., previously Commissioner and Senior Economic Advisor, Office of the Commissioner, respectively, at the U.S. Food and Drug Administration, without whose encouragement this project would not have been possible. We also thank the interviewees in industry, academia and the FDA who generously gave of their time to meet with us. Responsibility for any errors and opinions expressed herein rests solely with the authors. The authors can be reached at eberndt@mit.edu, agottschalk@sloan.mit.edu,mstrobec@ mit.edu.The views expressed herein are those of the author(s) and do not necessarily reflect the views of the National Bureau of Economic Research.

(C2005 by Ernst R. Berndt, Adrian H. B. Gottschalk, and Matthew W. Strobeck. All rights reserved. Short sections of text, not to exceed two paragraphs, may be quoted without explicit permission provided that full credit, including $($ notice, is given to the source. 
Opportunities for Improving the Drug Development Process: Results from a Survey of Industry and the FDA Ernst R. Berndt, Adrian H. B. Gottschalk, and Matthew W. Strobeck

NBER Working Paper No. 11425

June 2005

JEL No. I1, H11, K23

\section{$\underline{\text { ABSTRACT }}$}

In the United States, the Food and Drug Administration (FDA) agency is responsible for regulating the safety and efficacy of biopharmaceutical drug products. Furthermore, the FDA is tasked with speeding new medical innovations to market. These two missions create an inherent tension within the agency and between the agency and key stakeholders. Oftentimes, communications and interactions between regulated companies and the FDA suffer.

The focus of this research is on the interactions between the FDA and the biopharmaceutical companies that perform drug R\&D. To assess the current issues and state of communication and interaction between the FDA and industry, we carried out a survey of industry leadership in R\&D and regulatory positions as well as senior leadership at the FDA who have responsibility for drug evaluation and oversight.

Based on forty-nine industry and eight FDA interviews we conducted, we found that industry seeks additional structured and informal interactions with the FDA, especially during Phase II of development. Overall, industry placed greater value on additional communication than did the FDA. Furthermore, industry interviewees indicated that they were willing to pay PDUFA-like fees during clinical development to ensure that the FDA could hire additional, well-qualified staff to assist with protocol reviews and decision-making.

Based on our survey and discussions, we uncovered several thematic opportunities to improve interactions between the FDA and industry and to reduce clinical development times: 1) develop metrics and goals at the FDA for clinical development times in exchange for PDUFA like fees; 2) establish an oversight board consisting of industry, agency officials, and premier external scientists (possibly at NIH or CDC) to evaluate and audit retrospectively completed and terminated drug projects; and 3) construct a knowledge database that can simultaneously protect proprietary data while allowing sponsor companies to understand safety issues and problems of previously developed/failed drug programs.

While profound scientific and medical challenges face the FDA and industry, the first step to reducing development times and associated costs and facilitating innovation is to provide an efficient regulatory process that reduces unnecessary uncertainty and delays due to lack of communication and interaction.

\author{
Ernst R. Berndt \\ Sloan School of Management \\ MIT, E52-452 \\ 50 Memorial Drive \\ Cambridge, MA 02142 \\ and NBER \\ eberndt@mit.edu
}

$\begin{array}{ll}\text { Adrian H. B. Gottschalk } & \text { Matthew W. Strobeck } \\ \text { Sloan School of Management } & \text { MIT } \\ \text { MIT } & \text { mstrobec@mit.edu } \\ \text { gottschalk@sloan.mit.edu } & \end{array}$

Adrian H. B. Gottschalk

MIT 
"The FDA is responsible for protecting the public health by assuring the safety, efficacy and security of human and veterinary drugs, biological products, medical devices... and...for advancing the public health by helping to speed innovations that make medicines and foods more effective, safer and more affordable..."

From the FDA Mission Statement, (http://www.fda.gov/opacom/morechoices/mission.html)

"If biomedical science is to deliver on its promise, scientific creativity and effort must also be focused on improving the medical product development process itself, with the explicit goal of robust development pathways that are efficient and predictable and result in products that are safe, effective and available to patients. We must modernize the critical development path that leads from scientific discovery to the patient."

From Challenge and Opportunity on the Critical Path to New Medical Products, U.S. Department of Health and Human Services, Food and Drug Administration, March 2004, p. 5.

"Communication leads to increased collaboration, and increased collaboration leads to successful drug development."

Anonymous Interviewee, Global Head of Company R\&D

FDA Commissioner Mark McClellan believes “...that poor communication between the FDA and firms seeking drug approval adds months to reviews - and costs companies millions of dollars."

From Daniel Kadlec, “Will This Experiment Work?”, Time, 7 July 2003. 


\section{INTRODUCTION AND BACKGROUND}

Major scientific breakthroughs, new discovery technologies, and significant increases in life science $R \& D$ spending in both the private sector and at the National Institutes of Health have helped create prospects for the impending discovery and development of significant new medicines to treat unmet health needs. Despite these scientific advances and enhanced R\&D efforts, the number of average annual New Drug Applications ("NDAs") and new Biologic License Applications ("BLAs") approved by the US Food and Drug Administration has been smaller after 2000 than in the mid-1990s. ${ }^{3}$ Moreover, recent estimates suggest the average costs of bringing a new medicine to market have increased sharply to between $\$ 800$ million and $\$ 1.7$ billion, with the lower estimate being two and a half times higher than similar inflation-adjusted estimates published a dozen years earlier. ${ }^{4}$

This increase in costs of bringing new medicines to market may be somewhat surprising since, spurred by Congressional legislation, over the last decade review times at the FDA have actually been declining. Specifically, available evidence suggests that the passage and implementation of the Prescription Drug User Fee Act in 1992, and its successors in 1997 and 2002, have resulted in industry and the FDA working together to reduce mean and median NDA/BLA approval review times substantially, by more than $40 \%$ across a wide range of therapeutic classes. ${ }^{5}$

While the shortening of approval review times at the FDA has resulted in new drugs coming on to the U.S. market more rapidly, other things equal, this FDA review time comprises only a small proportion - about $10 \%$ to $15 \%$-- of the total time required to discover, develop and market a new medical product. 
The total time in drug development is typically broken down into pre-clinical and clinical stages. Prior to a sponsor filing an Investigational New Drug ("IND”) application with the FDA, authorizing clinical testing of a new compound for safety in healthy humans, sponsors typically engage in a pre-clinical drug discovery process that lasts from one to five years. Notably, the time between the IND authorization and the filing of the NDA/BLA is much more lengthy and, unlike the NDA/BLA review process, this interval has become longer rather than shorter. For example, one recent study reports that over the eight-year period 1994-2002, the average time elapsed between the IND and NDA/BLA filing was 76.7 months (about 6.4 years), virtually the same as the 77.3 month mean over the previous eight-year period from 1986-1994. In comparison, these more recent means are about a third larger than the 57.8 month (4.8 years) mean time interval between IND to NDA/BLA filing during the 1978-1986 time period, and more than $50 \%$ greater than that between 1970 and 1978. (49.2 months, or 4.1 years). ${ }^{6}$

What are the underlying factors leading to increased development times and costs? Why have the opportunities created by significant scientific and computational advances not yet resulted in greater numbers of successful and less costly new therapies being approved? How can industry and the FDA adapt or transform themselves to help make the drug development process - both pre-clinical and clinical -- more productive?

Given that drug development times are much longer than approval review times, and that the former have been increasing rather than falling in recent decades, it is clear that the drug development process merits a close examination. That is the focus of the research reported here, which is based on results from a 2004 survey of 57 senior R\&D and regulatory personnel in industry and at the FDA. 
The research reported here complements a recent FDA initiative. Specifically, in its March 2004 “Critical Path” document, Innovation or Stagnation: Challenge and Opportunity on the Critical Path to New Medical Products, the FDA considers a broad range of underlying scientific and manufacturing as well as clinical development issues for all types of medical products - pharmaceuticals, biologics, devices, diagnostics, etc. By comparison, here we focus our attention on clinical development efforts involving only pharmaceuticals and biologics (together called “drugs").

In particular, we report here the results of a recently completed confidential interview survey project assessing the nature of communications between the FDA and industry during the drug development process, and identifying opportunities to improve the management of this process. The survey results were compiled after conducting interviews with 49 senior R\&D/regulatory affairs officials at seven biotech/biopharmaceutical firms, seven pharmaceutical companies, and three contract research organizations (“CROs”). To obtain comparable perspectives from the FDA, we also conducted analogous interviews with eight senior FDA officials.

Two features of this survey deserve special note. First, the industry officials we interviewed were senior $\mathrm{R} \& \mathrm{D}$ and regulatory personnel, and in particular were not in the financial, marketing or public affairs divisions of these organizations; the views of these R\&D and regulatory personnel may differ considerably from managers elsewhere in these company. Second, all survey interviews were carried out between January 8, 2004 and May 27, 2004, at least five months prior to the September 30, 2004 voluntary withdrawal by Merck \& Co. of the Cox-2 inhibitor Vioxx, and the subsequent set of events involving FDA oversight of other pain killers, as well as hearings on the pediatric safety of antidepressant medications. 
The outline of this paper is as follows. In Section II we provide a brief summary of the clinical drug development process, and focus on significant milestones and FDA-industry interactions during this process. In Section III we describe our research methods, while in Section IV we discuss ten sets of survey findings. We comment on related qualitative and "thinking outside the box" de novo responses further in Section V, summarize themes in FDA and industry interviewees' recommendations in Section VI, and note study limitations in Section VII. A more detailed quantitative analysis of our findings, along with a set of appendixes providing further details concerning study design and research methods, are contained in a preliminary draft version of this paper, available upon request from the authors. ${ }^{7}$

\section{BRIEF OVERVIEW OF THE DRUG DEVELOPMENT PROCESS}

The process of basic discovery through new drug approval consists of pre-clinical and clinical development. Its structure, along with typical timelines and a recent estimate of conditional transition and cumulative attrition rates, is summarized in Figure $1 .^{8}$ The preclinical portion of development begins with basic discovery and research and extends through animal testing. Early portions of pre-clinical development consist of scientific in vitro and in vivo experiments and validation of principles and concepts; such research takes place within academic, government and industry laboratories. Generally a lead or candidate compound is first identified and isolated after screening thousands of chemicals/proteins against a specific biological target. Next safety/toxicity animal studies are conducted with this compound. After carrying out extensive pharmacokinetic and pharmacodynamic testing in various animal models, the developing company, known as the sponsor, can file an Investigational New Drug ("IND”) application, which must clear the FDA before human testing can commence in the U.S. The 
length of this pre-clinical development process is highly variable, but it typically lasts between one and five years. ${ }^{9}$

FIGURE 1: DURATION AND TRANSITION PROBABILITIES OF DRUG DEVELOPMENT PHASES

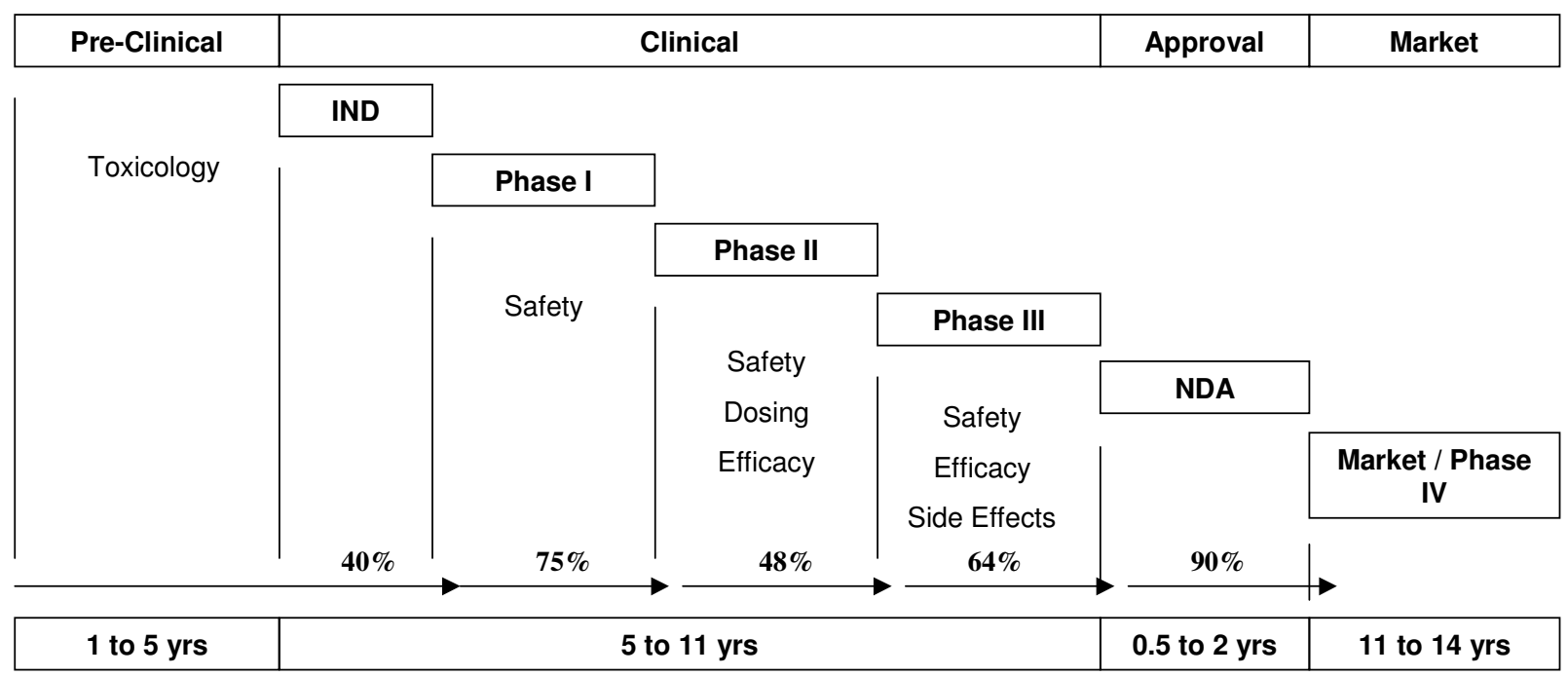

Once an IND application clears the FDA, a sponsor can initiate clinical studies in humans. Approximately $40 \%$ of INDs transition to Phase I trials. ${ }^{10}$ Phase I clinical trials are designed primarily to test for safety and tolerability of the drug in humans through the generation of pharmacokinetic data involving the absorption, distribution, metabolism and excretion of the drug. This phase usually involves a small group of healthy, nominally paid volunteers, numbering from 20 to 100 individuals, and lasts between one and three months. Approximately $75 \%$ of compounds transition from Phase I into Phase II.

In Phase II, the preliminary effectiveness of the candidate drug is assessed, as is safety and tolerability via continued monitoring within dose ranges established in the Phase I studies. Phase II trials often involve several hundred unpaid volunteers diagnosed with a particular 
illness/condition, and typically take from six months to two years to complete. Slightly less than $50 \%$ of NMEs tested in Phase II proceed into Phase III.

Phase III trials, often called pivotal clinical trials, are designed to evaluate statistically the safety and efficacy of the drug compared to placebo or standard of care within a larger and typically more diverse population. These trials involve hundreds to several thousand patients (depending in part on the therapeutic area, and whether the drug treats an acute or chronic condition), and often include examination of alternative formulations and doses of the drug. Due to these characteristics, the Phase III trials are the most costly stage of drug development. In most cases the sponsor conducts several Phase III trials, with the average length of time of the entire Phase III process being about four years. It is estimated that about $64 \%$ of drugs tested in Phase III trials transition successfully into NDAs or BLAs. ${ }^{11}$

Once submitted to the FDA for review, approximately $90 \%$ of NDAs/BLAs eventually receive FDA approval and are marketed. Review times now average about one year. When the various between-phase and final approval transition probabilities are multiplied to achieve a cumulative probability, the chance of a leading drug candidate successfully proceeding from preclinical to approval is about $8 \%$; for every $12-13$ compounds that were serious candidates in preclinical research, only one drug will make it to market.

Phase IV trials, also known as post-marketing studies, are in some cases performed as a condition required by the FDA for initial market approval. In other cases they are undertaken to obtain approval for a new indication, or are carried out for marketing purposes. Phase IV studies are designed to observe the (sometimes long-term) effects of a drug in a larger and more heterogeneous population than studied in the Phase III trials. It is not unusual for Phase IV studies to involve thousands of patients; on average, these studies take three to four years, though 
for some chronic conditions, they can last much longer. Once on the market, new patentprotected drugs typically have 11 to 13 years of market exclusivity before facing generic competition, although they likely face therapeutic competition much earlier.

Because of the long development process (about 7.5 years on average from IND filing to final NDA/BLA approval), a substantial portion of drug development costs involves the opportunity cost of capital, i.e., earnings the sponsor could have realized had it instead invested funds elsewhere. For example, in the DiMasi, Hansen and Grabowski [2003] study cited earlier, of the $\$ 802$ million average cost of bringing a drug to market, almost exactly half ( $\$ 403$ million) consisted of direct out-of-pocket costs, while the remainder reflected opportunity costs, capitalized at an $11 \%$ annual real discount rate. One recent study has reported that if the probability of successfully transitioning from Phase I to market approval improved by a factor of one-half (from $21.5 \%$ to $33 \%$ ), capitalized costs per drug would be reduced by about $30 \%$ (from $\$ 802$ to $\$ 560 \mathrm{MM}$, or $\$ 242 \mathrm{MM})$. In comparison, a reduction of $50 \%$ in out-of-pocket costs across all clinical development phases would have virtually the same effect, reducing capitalized costs by $\$ 235 \mathrm{MM} .^{12}$ Simply stated, time is money, and the longer the development time for drugs, the greater the capital that is invested cumulatively. Efforts that result in reduced preclinical and clinical development times are therefore likely to be particularly valuable.

To understand the management issues encountered in the course of drug development, it is useful to depict industry-FDA interactions along the pathway involving various pre-clinical and clinical phases of the drug development process. Figure 2, taken from the FDA's March 2004 paper, Innovation or Stagnation? Challenge and Opportunity on the Critical Path to New Medical Products, provides one characterization of the most common industry-FDA interactions. Sponsors often meet with the agency before submitting an IND to discuss early development 
plans. During the clinical phases, there are ongoing submissions of new protocols, and discussions of preliminary results from testing. Among the multiple informal and formal interactions between the sponsor and the FDA, there are three critical meetings. One of the key meetings is called the end of Phase II-A meeting, during which preliminary findings involving dose and safety are discussed, as well as future clinical protocols and endpoints. Another key

FIGURE 2: INDUSTRY-FDA INTERACTIONS DURING DRUG DEVELOPMENT

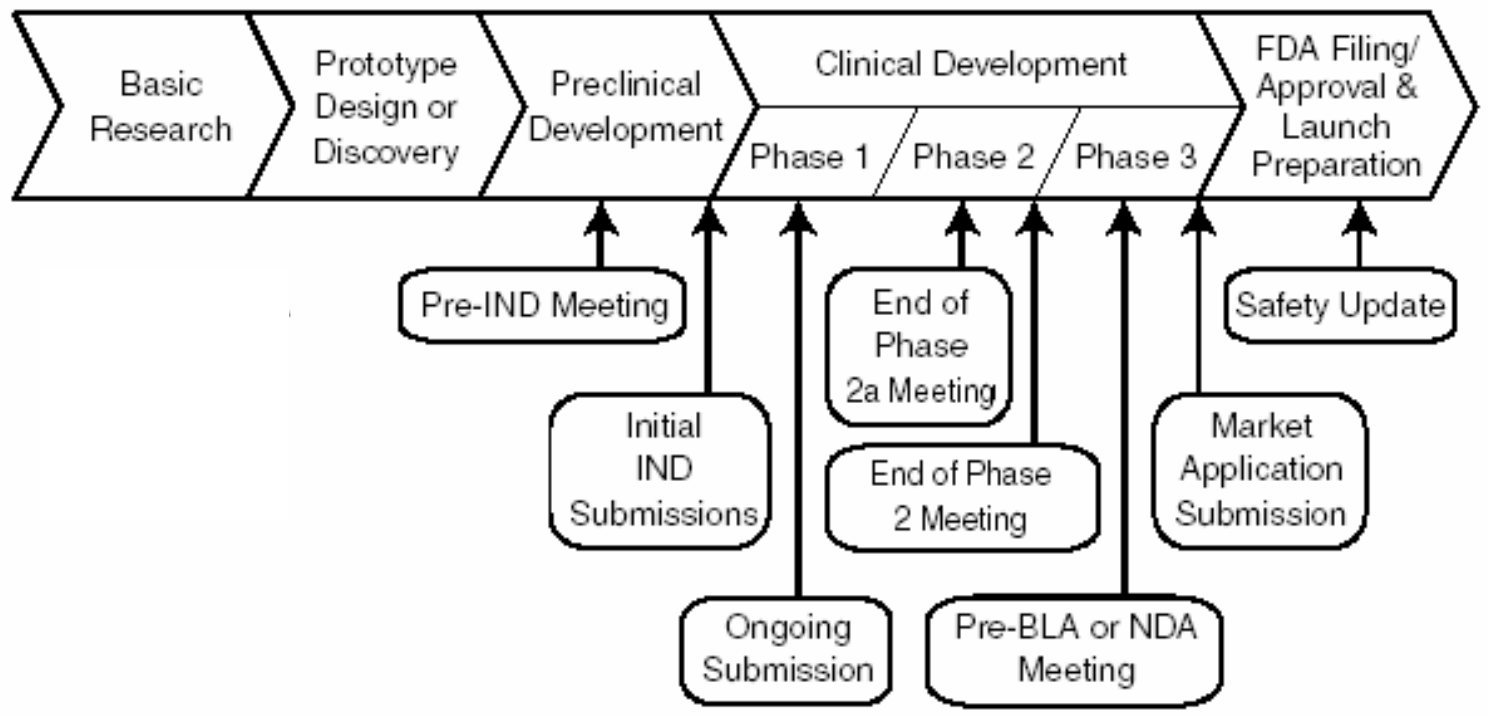

meeting is the end of Phase II meeting ("Phase II-B"), in which detailed plans are discussed for the design of the pivotal clinical trials, and agreement is sought on methods and endpoints proposed for the evaluation of safety and efficacy. A third key meeting is the pre-BLA/NDA submission meeting, in which sponsors meet with medical reviewers and other FDA officials to discuss the clinical package about to be filed.

III. RESEARCH METHODS

A series of 49 interviews each involving at least two of the three coauthors was undertaken with a total of 17 drug developing companies that had locations in the United States. 
These interviews, conducted between January 8, 2004 and April 29, 2004, involved seven medium to large biotechnology/biopharmaceutical firms, seven very large pharmaceutical companies, and three contract research organizations (one of them privately held). All of the pharmaceutical and biotechnology/biopharmaceutical firms are public companies, and all but one has at least one product on the market. The total market capitalization of the 17 public companies as of April 2004 was more than a trillion dollars. Interviews with a total of eight senior FDA officials were conducted by at least two of the three coauthors on May 4, 2004 and May 27, 2004.

For the industry interviews, an attempt was made to identify individuals at senior level positions within their company and who had significant responsibilities for pre-clinical development, clinical development, regulatory oversight, or combinations of these activities. Of the 49 individuals interviewed, 88\% held positions of Vice-President, Executive Vice-President, or Global Head of R\&D or Regulatory Affairs. Twenty-two individuals were interviewed from pharmaceutical companies, twenty-one from biotech/biopharmaceutical companies, and six from CROs. For the FDA, interviews were conducted with eight individuals, each currently a division head or higher, and all having experiences across multiple FDA offices or divisions.

Both industry and FDA interviews involved quantitative and qualitative aspects. The initial portion of each interview involved asking the interviewee to rank responses to a series of statements or questions on a scale of one to five. Respondents identified the issues to which they wished to return, to discuss them in greater depth. In the qualitative portion of the interview, the interviewee was encouraged to elaborate on the earlier quantitative responses of particular interest. Industry interviews typically lasted 60-90 minutes, while those at the FDA were all approximately 60 minutes long. 


\section{RESULTS FROM THE SURVEY}

We now summarize the ten most common and striking findings from our survey, primarily in a qualitative fashion; a more detailed and quantitative discussion is found elsewhere. $^{13}$

\section{A. INDUSTRY HAS GUARDED RESPECT FOR THE FDA}

At the beginning of each company interview, we asked interviewees to rate the agency's ability to regulate drug development and appropriately weigh the risks and benefits of new drugs. Industry gave the FDA an average rating of "good", a rating that did not vary significantly across biotech, pharmaceutical and CRO respondents. Qualitative comments from interviewees indicated a great deal of respect for the FDA as a whole and recognition of the challenging role the agency plays in evaluating new drugs.

Although industry's average rating of the FDA's effectiveness at keeping unsafe drugs from the market was even higher (between "good" and "excellent"), follow-on questioning resulted in many respondents qualifying their answers to this question with the comment that the agency was partly responsible for delaying the progress of some very valuable new therapies to market, in some cases being overly vigilant. One global head of R\&D stated the agency tended inappropriately to weigh drug development as a "risk-benefit" analysis. Instead, the individual argued, a "risk-risk" analysis would often be more appropriate, since the tradeoff frequently involves comparing the risks of approving a drug having certain safety issues with the risks that patients face without having the therapeutic available as a treatment option. This individual also stated that often it is the risk of the drug being used in inappropriate populations or combination therapies that causes safety concerns and delays at the FDA. 


\section{B. PDUFA HAS HAD A SIGNIFICANT BENEFICIAL IMPACT ON APPROVAL TIMES, BUT OTHER TRENDS ARE WORRISOME TO INDUSTRY}

Industry interviewees agreed for the most part that the FDA had made significant efforts to reduce drug approval times, but that the amount and impact of efforts by the FDA to reduce clinical development times were modest at best. Responses from the FDA interviewees were qualitatively similar to those from industry.

FDA interviewees acknowledged that in terms of affecting review approval times, PDUFA had brought about a huge cultural shift at the FDA. As one FDA official put it, "The review process is now a Swiss train, not an Italian train." However, a number of agency officials also acknowledged that the focus on reducing clinical development times was still not universally shared, particularly among reviewing staff. For example, one senior official noted that for decades facilitating reductions in clinical development times had not been perceived as a priority at the FDA, nor perhaps even a role the FDA ought to play. Instead, the traditional attitude of reviewers has been one of asking for whatever data is necessary, without much concern to time delays and costs imposed on the sponsor. Attention to the second mission of the FDA - promoting the public health by helping to speed access to new medical innovations - was a relatively recent phenomenon and less universally shared, said another official. On the other hand, both FDA and industry personnel noted that the FDA's recent record on issuing guidance documents was improving, and that these documents (as well as meetings leading up to them) helped reduce uncertainties in the drug development process. Several interviewees specifically identified a recent FDA guidance document involving pediatric clinical investigations as being exemplary. 
When asked whether the European Medicines Evaluation Agency ("EMEA") was more efficient than the FDA in approving drugs, about two-thirds of industry interviewees either disagreed or were neutral. One "best practice" of the EMEA cited by several interviewees was its convening of an expert meeting of regulators, sponsors and academics whenever significant developments in a therapeutic area or treatment modality had taken place.

A number of industry interviewees commented that historically, a strength of the FDA has been that its mandate has been limited to evaluating medicines on the basis of scientific criteria, and not on the basis of commercial or reimbursement considerations. Companies' experiences with the EMEA and national health authorities have historically been less satisfactory. Specifically, in Europe both reimbursement and approval decisions were often more deeply intertwined. Others believed that the EMEA's stand on wanting comparative trials (not just placebo controls) was unfortunate, for that implicitly introduced cost and price concerns, rather than a focus on scientific issues involving efficacy. Several interviewees voiced concerns that with passage of the Medicare Drug Benefit legislation and increased collaboration between the FDA and the Centers for Medicare and Medicaid Services, this separation of mandates could become more blurred in the U.S. as well.

\section{EXCESSIVE VARIABILITY ACROSS DIVISIONS AT THE FDA}

In almost every company interview, respondents indicated that there was a high degree of variability in competence, communications and implementation of rules and regulations across divisions at the FDA. A substantial number of respondents characterized the FDA as being organized like a "cottage industry", where divisions are relatively independent, interacting in highly variable ways with sponsors. 
While acknowledging that various divisions in the Center for Drug Evaluation and Research ("CDER") are engaged in different therapeutic areas and thus would reasonably be expected to have different requirements for establishing safety and efficacy, company interviewees repeatedly stated that the ways and extent to which divisions interact and respond to sponsors is highly variable, from simple matters such as returning phone calls to more complex issues such as feedback on clinical protocols and NDAs/BLAs.

Several divisions were consistently identified by industry as having "best practices" that accelerated the drug development process and made it more predictable. These best practices included the following:

- rapid turnaround of agreement on meeting minutes (in some cases before the end of the meeting, projected onto a screen visible by all);

- invitation by the FDA to the sponsor to make a half-day presentation to the therapeutic division on a novel drug, including a discussion of the underlying science and outcome metrics; and

- implementation of an open communication policy, including FDA commitment of within 24-hour acknowledgement of sponsor phone call.

However, several other divisions were identified by interviewees as having "worst practices". Such practices included:

- very poor communication protocols (only willing to discuss issues via letters, and not the telephone);

- extended time delay in resolving issues relative to FDA-sponsor commitments, including multiple changes in previously agreed upon decisions between the sponsor and the FDA; 
- ambiguous advice and unwillingness to commit to protocols; and

- preoccupation with minor statistical issues essentially unrelated to therapy evaluation, e.g., a patient's bowel surgery while on an antidepressant.

Agency officials are very much aware of variability in communications protocols across divisions. When asked how sponsor companies would rate the consistency of communications and interactions across therapeutic divisions, FDA interviewees rated these as on average being slightly above poor. One senior agency official stated that an outside consultant had recently been retained to address how best practices could be defined across the FDA's divisions, and what metrics could be put in place to monitor progress. In an initial attempt to address these issues explicitly, in October 2003 the FDA issued a draft guidance document on Good Review Management Principles, designed for both industry and FDA staff. At the time of our interviews in May 2004, apparently this document was in the process of being revised. As an aside, this individual also noted some disagreement with the above-named best practice of the FDA and industry agreeing on minutes at the end of meetings, and perhaps even projecting them on a screen for all to see. One potential problem with that, this individual argued, was that the minutes might be unable adequately to explain why a particular decision had been made. The underlying rationale was often important, and if not memorialized in minutes, with fading memories its absence could complicate downstream negotiations and decision-making, as well as obfuscate applicability to other development projects.

Biotech companies in particular expressed concern over the merger of CBER and CDER, noting that CBER had established a track record of engaging in many of the best practices (quick response to sponsor inquiries, accessibility of reviewers and leadership, proactive interest in the 
underlying science) which they feared might not be sustained under the auspices of an augmented CDER.

\section{MIXED VIEWS ON APPROPRIATE TRAINING OF MEDICAL REVIEWERS}

Industry respondents rated the training of medical reviewers after joining the agency (not their previous education or training) as generally being "fair" to "poor". Follow-on questions revealed that in fact industry has relatively little knowledge of the on-the-job and other formal training FDA medical reviewers receive, although several interviewees indicated they had made presentations at an "FDA campus". Numerous company interviewees acknowledged that the FDA medical reviewers have a very difficult job, that many work long hours and likely are underpaid relative to industry employees.

In comparison, FDA respondents rated the ongoing training of their medical reviewers much higher, being on average "good". However, follow-on discussion with FDA officials revealed that while the scientific training of reviewers was generally of high quality, in most cases they had little if any management training. One FDA official noted wryly that since so many of the reviewers were trained as physicians and/or academics, it should not be surprising that their "people skill" and "management skill" sets may be lacking, and that industry might understandably assess them as being of low quality on these dimensions. Another FDA interviewee noted that as part of their training new medical reviewers are required to attend special classes, including courses that focus on critical writing and communication skills, but that most of the learning is still "in-service" or on-the-job training. One division director indicated with satisfaction the use of an annual "West Virginia retreat" that emphasized people skill development, not science education. A strong mentoring program would be most helpful, stated another agency individual, augmented with some formal management education. Citing 
previous efforts that resulted in "reviewers impervious to management training," one agency official also pointed out that care is needed to ensure that new recruits are not so far along in their careers to be unable to change their working attitudes and habits - particularly if they are physicians.

Differences between industry and FDA officials' perceptions also emerged in response to a related question assessing the quality of FDA reviewers. While the average industry response was in between "fair" and "good", the FDA average rating was significantly higher, between "good" and "excellent".

However, when asked to evaluate the FDA leadership (team leader, deputy division director, and division director) of medical reviewers, industry respondents ranked the leadership considerably higher than the medical reviewers, on average between "fair" and "good". Followon questioning revealed that company interactions with FDA leadership one or more levels above the medical reviewer were generally positive. Industry respondents explained that excellent medical reviewers were likely to become team leaders and move up through the FDA hierarchy, generating higher ratings for the leadership. Another industry official pointed out that over the last five years the FDA had made significant efforts in hiring qualified senior leadership. As an example, the individual noted that just several years ago the FDA's CBER office hired a distinguished imaging specialist, who has since been moved to CDER; no previous radiopharmaceutical division head had ever been a radiologist.

Industry interviewees expressed significant concern over the turnover rate of medical reviewers at the FDA (particularly in toxicology), and while unable to cite quantitative evidence, a number suggested that turnover at the FDA was higher than in industry for positions of equal responsibility. More importantly, upon turnover of a medical reviewer, companies were often 
required to revisit numerous previous decisions that had been agreed upon with the agency, causing unnecessary delays (although in some other cases, speeding up reviews when a very senior reviewer with idiosyncratic scientific views finally retired). Several respondents suggested that a formal handoff procedure, involving participation by both FDA reviewers and sponsor, take place whenever a turnover occurs at a critical review level. Companies also acknowledged, however, that in some cases turnover within their regulatory group also delayed drug development. ${ }^{14}$ FDA interviewees indicated that in their view turnover at the medical reviewer level had been decreasing, unlike that at the more senior division director level.

\section{E. TENSIONS EXIST WITHIN COMPANIES ON STRATEGIES FOR DEALING WITH THE FDA}

Pharmaceutical and biotechnology companies were asked how well they were organized to deal with the FDA. While both biotech and pharmaceutical companies envisaged themselves as on average being "good" in this respect, biotech's self-perceptions were slightly higher than those by pharmaceutical respondents.

Follow-on discussion revealed that an elaborate regulatory group is typically set up within each company, acting as the primary link between the rest of the company and the FDA. The majority of interviewees indicated that this structure usually works reasonably well. However, several interviewees noted that pressures from general management and marketing occasionally forced the regulatory group into confrontational situations with the FDA.

Moreover, a substantial number acknowledged that mergers or acquisitions had made interacting with the FDA more difficult, in part because of inconsistent regulatory practices, and different histories and cultures among the new partners in how aggressive or accommodating they should be with the FDA, resulting in delayed internal decision-making. FDA officials concurred, 
indicating that in their experience, mergers and acquisitions often led to loss of some of the best scientists, disagreements on dosing, labeling and other clinical strategies previously employed by the new partners, and confusion and uncertainty resulting in delayed communications with the FDA.

\section{F. DIVERSITY IN CONFRONTING AND DISAGREEING WITH THE FDA}

Industry officials had bimodal responses to questions regarding whether companies were fearful of disagreeing with the agency on protocols; roughly equal numbers "agreed" their company was afraid to push back, or either "disagreed" or "strongly disagreed". Pharmaceutical company interviewees were more likely to agree that their regulatory groups were hesitant to confront the agency, whereas biotech companies indicated they were less fearful to push back on the FDA regarding clinical protocol requirements, although this difference was not quite statistically significant (p-value of no difference, 0.095).

\section{G. INSUFFICIENT DOSING ANALYSES IN PHASE II TRIALS COMMON}

Bimodal responses also occurred in regards to whether companies ran additional clinical trials not required by but in anticipation of questions the FDA might ask. Biotech companies were much less likely to run such additional trials, whereas pharmaceutical companies indicated they did. Follow-on discussion revealed that in many cases companies ran additional trials for marketing and/or labeling purposes (including quality of life analyses). While in a strict sense a few of these trials were unnecessary for the approval of the drug based on FDA requirements, some companies indicated they have tended to run additional Phase II trials to ensure they had identified the proper dose effective range, thereby reducing downside risks associated with subsequent Phase III trials. 
A substantial number of company interviewees stated that failure to run a sufficient number of Phase II trials to detect the appropriate dose range had been instrumental in causing failures in Phase III. Some acknowledged they had witnessed development programs within their company that progressed too rapidly through Phase II in order to get to Phase III, perhaps due to demands and pressures from the investment community to show progress in reaching the next targeted development milestone.

Many of the issues raised by industry were also voiced by FDA officials, particularly those involving rather hurried Phase II trials and insufficiently extensive dosing analyses. Several FDA interviewees identified the industry's tendency to focus excessively on once daily dosing regimens, for in some cases that simply was unlikely to be efficacious; in one failed Phase III study with a once-daily dosing, for example, the half-life of the drug was only four hours, and the drug was cleared before exerting any significant therapeutic effect.

\section{H. VALUATIONS OF EXISTING AND ADDITIONAL COMMUNICATIONS WITH THE FDA VARY SYSTEMATICALLY EXCEPT IN PHASE II}

Companies were asked to rate the quality of their current communications with the FDA during the various development phases, and their valuations of additional communications. A striking set of findings was the uniformly high valuation by industry and the FDA of additional Phase II informal communications, but systematic discordant FDA-industry valuations of such additional communications during the other drug development phases. Industry and FDA valuations of the quality of current state of communications was generally higher during the later development phases than in earlier stages.

Biotech companies on average rated the current pre-clinical communications with the FDA as being "good", but pharmaceutical companies rated their pre-clinical communications 
significantly lower, slightly less than "fair". A similar pattern of responses emerged for quality of communications during Phase I. However, FDA interviewees consistently rated the quality of their communications with industry sponsors during the pre-clinical and Phase I stages more favorably than did industry.

The assessments of quality of current communications between sponsors and the FDA were much more uniform for Phase II, but this uniform rating was not a stellar one. Both biotech and pharmaceutical companies rated the quality of their current interactions during Phase II as being on average between fair and good, as did the FDA. A consistent theme in cases where industry rated communications as "fair" was the perceived variability in communications, ranging from extremely poor to excellent, across the FDA's therapeutic divisions. Follow-on discussion with FDA interviewees suggested that the FDA was aware of this variability in communications quality with industry across the FDA's divisions during the early development phases. One FDA official noted that the FDA had relatively little experience in the discovery stage, although it has about 250 pre-IND meetings annually with sponsors.

Viewed from both industry and FDA perspectives, the quality of current communications between industry and the FDA was generally higher during the later development phases than in earlier stages. During Phase III, both the FDA and overall industry rated the quality of these communications as being close to "good"; similarly, FDA and overall industry perceptions were that the quality of communications during the NDA/BLA review stage was "good". .

To examine the importance of milestone meetings with the FDA, companies were asked to rate the value of the consultation at the end of Phase II, prior to Phase III. At this meeting decisions are often made on scoping out final details for the design of and endpoints used in the 
pivotal Phase III trials. Biotech and pharmaceutical respondents uniformly rated this consultation as being on average close to "very valuable".

Notably, while both pharmaceutical and biotech companies find the end of Phase II meeting to be very valuable, earlier we reported they also rate the quality of current interactions during Phase II as only on average being “fair". Follow-on discussion suggested that industry believed that significant opportunities exist for higher quality communications during this key drug development phase. Some industry interviewees suggested that instituting a user fee program analogous to PDUFA for early clinical studies might be useful, although perhaps not all the way back to the IND or Phase I stages, particularly since a considerable number of INDs are filed by individuals and/or non-profit organizations.

Company and FDA interviewees were asked how valuable additional informal communications would be in the various phases of drug development. With little variability among them, companies rated such communications extremely highly -- being between "valuable" and "very valuable" during the pre-clinical and all the clinical phases, as well as during the NDA/BLA review process. One global head of R\&D summarized industry's view succinctly, saying "Communication leads to increased collaboration, and increased collaboration leads to successful drug development."

By comparison, in four of the five stages, FDA interviewees rated the value of such additional informal communications with sponsors as significantly lower than did industry - preclinical, Phase I, Phase III and NDA/BLA review. In each of these four stages, while industry valued additional informal communications with the FDA as being on average between "valuable" and "very valuable", mean FDA valuations were either "ambivalent" or in between "ambivalent" and "valuable". 
However, a striking result is that only during Phase II do industry's and the FDA's valuations of increased informal communications match each other - both rating these between "valuable" and "very valuable". Notably, FDA interviewees apparently believe that the value of additional informal communications is much higher in Phase II than during other drug development stages. Follow-on discussion with FDA interviewees revealed their belief that Phase II dosing issues were critical, and that a more formalized and extensive Phase IIA meeting, scheduled at the time the first data on dosing become available, would be very useful.

The FDA's experience is that at this stage of the drug development process, there is frequently a tension between the sponsor's "academic science" and "commercial interests", and that a more formalized discussion and review of initial pharmacokinetic and dosing data could help to clarify development issues. At that meeting, FDA officials envisaged an increasingly important role for clinical pharmacological analyses. Waiting until the end of the Phase II studies was often too late, they argued, for by then sponsors had often made up their minds on dosing ranges for the subsequent Phase III pivotal studies. Another FDA interviewee noted that currently a Phase IIA guidance paper was being drafted and discussed with industry, which it was hoped would help sponsors deal more preemptively with dosing issues.

Our survey indicated further that industry was willing to put its money where its mouth is. Specifically, to assess the strength of their desire for increased communications, industry interviewees were asked whether their companies would be willing to pay more for communication with the FDA during various development phases to help the FDA strengthen and expedite clinical development reviews, and to facilitate discussions on various regulatory issues by hiring additional staff. For Phase I, $70 \%$ of respondents indicated they would be willing to pay PDUFA types fees ranging between $\$ 100 \mathrm{~K}$ and $\$ 500 \mathrm{~K}$, while $30 \%$ would be 
willing to pay between $\$ 500 \mathrm{~K}$ and $\$ 1$ million. Although $10 \%$ of respondents were unwilling to pay any PDUFA-type fees during Phase II, about $40 \%$ were willing to pay between $\$ 100 \mathrm{~K}$ and $\$ 500 \mathrm{~K}$, another $40 \%$ were willing to pay between $\$ 500 \mathrm{~K}$ and $\$ 1$ million, and $10 \%$ were willing to pay more than $\$ 1$ million. Finally, while $30 \%$ of respondents were unwilling to pay and PDUFA-type fees for Phase III, 40\% were willing to pay between $\$ 100 \mathrm{~K}$ and $\$ 1$ million, and $30 \%$ were willing to pay between $\$ 1$ million and $\$ 5$ million. In none of the three phases was there any statistically significant difference between pharmaceutical and biotech companies, although during Phase III pharmaceutical company willingness to pay tended to be greater than that by biotech firms.

These findings on the willingness of both biotech and pharmaceutical companies to pay additional user fees underscore the industry's perceived importance of participating in additional quality communications with the FDA. In some instances, however, follow-on discussion revealed that certain companies were hesitant to commit to paying additional user fees unless they could be assured any incremental funds would in fact be used to hire additional staff devoted to improving communications between the FDA and sponsor, and that metrics would be put in place to measure and monitor the incremental hiring and review efforts.

Although on average FDA officials valued additional informal communications in the early development phases less highly than did industry, several FDA interviewees believed there were substantial differences across companies in the likely benefits of such additional communications, with small and less experienced companies being most likely to benefit. One interviewee stated that "Pfizer doesn't need it", and then added, "Merck has three people who have been to more FDA meetings than I have." 
I. INDUSTRY CAUTIOUS REGARDING DEVELOPMENT AND VALIDATION OF BIOMARKERS AND SURROGATE MARKERS

The FDA's March 2004 "Critical Pathways" document argued that "The appearance of new quantitative measuring technologies absolutely galvanizes new drug research.” The document then went on to state: "Additional biomarkers (quantitative measures of biological effects that provide informative links between mechanism of action and clinical effectiveness) and additional surrogate markers (quantitative measures that can predict effectiveness) are needed to guide product development." 15

While industry interviewees exhibited some enthusiasm for increased use and development of surrogate markers, we learned that their outlook was tempered with considerable caution. A substantial number indicated that without appropriate guidance from the FDA, use of new surrogate markers for primary efficacy endpoints was simply too risky an undertaking.

Industry interviewees had a bimodal response to whether their company would be willing to use surrogate markers under current FDA guidelines, with 50\% indicating they were "somewhat likely" or "very likely" vs. 50\% being "ambivalent", "not likely" or "very unlikely". In cases where interviewees indicated they were "very likely" to use a surrogate marker, they typically also stated that they were attempting to validate the surrogate marker simultaneously with their ongoing clinical trials, or were employing a marker previously "accepted" by the FDA (e.g., CD4+ T-cell counts for AIDS).

Given the challenges in validating surrogate markers, we then inquired whether economic incentives, such as that from patent protection, would induce companies to engage in additional development and validation of surrogate markers. While the average response was essentially "somewhat likely", this was the only question where we observed statistically significant 
differences by interviewee rank; the more senior management (executive vice presidents and higher) believed their company would be less likely to employ surrogate markers if patent protection were available, whereas those with rank of vice-president or lower stated their company would be more likely to do so. Interestingly, while CRO interviewee responses (which we classified separately from industry and FDA) were generally indistinguishable from those of industry on most issues, each of the (admittedly small number of) CRO interviewees indicated they would be "very likely" to employ and validate surrogate markers were patent opportunities available.

Follow-on discussion diverged widely. Several interviewees in both pharmaceutical and biotech companies stated that it would not be in the interests of the research community, public health or even in the long-term interest of their company if surrogate markers could be patented, for that could impede use of critical research tools in their subsequent drug development efforts. Rather than patenting the marker, a number of interviewees suggested an extension of market exclusivity (such as that granted for pediatric indications), in exchange for placing the biomarker in the public domain, freely available for use by other researchers and developers.

Additional discussion indicated great hesitancy on the part of interviewees to rely on surrogate markers given current FDA guidelines and practices. While numerous interviewees indicated that biomarkers were extensively and increasingly used within their company to evaluate safety concerns or to assist in "go/no go" decisions, in most development programs traditional and already-accepted clinical endpoints still trumped biomarkers as efficacy and safety endpoints. 


\section{J. DISAGREEMENT ON THE VALUE OF FDA ADVISORY BOARD PANEL} MEETINGS

FDA division directors appoint advisory board members in different therapeutic areas to assist them in decision-making. The most public context in which advisory panels provide advice to the FDA is in the final stages of the NDA/BLA review process, after the FDA has had time to review and assess data from the application. Advisory board panel meetings are public and typically webcast. In most cases the sponsor initially makes a presentation, next the FDA summarizes its analyses, patients' advocacy groups make testimonials, advisory board panel members ask questions and finally, the advisory board panel votes on whether to recommend to the FDA that the application be approved. In most but not all cases, the FDA agrees with the panel's recommendations. Notably, while the FDA advisory board panels are not involved in the clinical development component of drug development, they do in fact play a key role in the drug approval process.

Industry is sharply divided in its valuation of this advisory process and panel meeting. On average, industry was "ambivalent" in its evaluation, with there being no systematic differences among pharmaceutical and biotech respondents. However, interviewees at the FDA rated this process much more favorably, on average in between "valuable" and "very valuable". CROs tended to agree more with the FDA than with industry on this issue.

Proponents of the advisory panel process indicated that it was a critical point in the NDA/BLA process that allowed the public and practitioner physicians to evaluate the drug, as well as witness the FDA at work. Detractors were critical of the advisory board process in no uncertain terms. Several interviewees likened the process with colorful descriptors as such "circus", "crap shoot" and "dog and pony show." Behind their colorful rhetoric, however, 
interviewees expressed great concern over the confrontational nature of the hearings. According to several companies that have had very successful approval hearings, yet are sharply critical of the process, the meeting is set up with the sponsoring company on one side, the FDA on the opposite side, and the advisory board as the adjudicator. Some interviewees noted that in communications with FDA officials prior to the panel meeting, the FDA voiced generally favorable evaluations, yet at the public hearing the FDA voiced primarily antagonistic views.

Industry interviewees indicated that substantial amounts of money and time are spent preparing for the "show." One company said that in preparation for this meeting, it prepared 1000 backup slides, while another claimed the number to be 1200 . These meeting preparations are highly labor-intensive. While agreeing that the public is entitled to learn about the NDA/BLA details given current Federal regulations (although also acknowledging that what is publicly disclosed at a meeting attended by competitors raises strategic issues for sponsors), and that it is important the public see the FDA at work, industry officials often argued that the current process does not do proper service to the sponsor or the FDA.

Several interviewees, who previously were high-level FDA personnel prior to joining industry, stated that panel experts are often poorly prepared for the review, "reading the material on the plane ride to the FDA." These respondents argued that given that the review process takes many FDA personnel several months, it is improbable that a panelist could gain sufficient understanding to render an appropriately informed decision after only a few hours of review time.

A substantial portion of industry respondents voiced concern over the qualifications of advisory panel members, indicating that more qualified experts were often excluded due to implementation of "excessively restrictive" conflict of interest regulations. Several interviewees 
stated that the panel meeting environment brought out some of the worst type of behavior by academic panel members, enabling them to "toot their horn and spout off useless remarks."

From the perspective of several industry respondents, the FDA was eminently wellqualified to make the decision on whether to approve the NDA/BLA, following its typically extensive and thorough review of all the safety and efficacy data, and that advisory board panel meetings were unnecessary. When controversial issues emerge, they argued, a better way of managing disagreements or uncertainties would be to have another government agency such as the NIH convene a forum attended by leading experts (even those with company affiliations), the FDA, industry, and academic personnel. It was not uncommon for these types of discussions to move to the more general qualitative issues of how dispute resolution could be better handled at the FDA. Later on we comment on this issue further.

While on average FDA interviewees viewed the advisory board panel process more favorably than industry, agency officials were aware of problems, and had some suggestions for improvement. First, for the meetings to be constructive, considerable work was required by FDA staff, a staff already stressed by what one official called a "Meetings R Us" mentality, with more than 1300 meetings annually between divisions and sponsors. Another FDA interviewee opined that the advisory board panel meetings are sometimes helpful, sometimes not, and then argued that decisions regarding approval often are more nuanced that just simply involving safety and efficacy. In particular, approval decisions often concern detailed regulatory issues such as labeling considerations, for which panel members typically have little experience or training. These issues should be addressable, but considerable care must be given in choosing panel board members, for their clinical experience provides a valuable perspective in assessing potential benefits and risks of using the new drug. Another FDA interviewee agreed, stating "It 
all depends on how well you select your participants." Yet another agency official suggested using advisory board panels only for selected applications, where their input is clearly needed.

Finally, a common theme that emerged from follow-on discussions with industry was that these advisory board panel meetings provided the FDA and industry a unique opportunity to educate the public (and the FDA staff) on the importance of risk management in the drug regulatory process - not just the safety and toxicity risks from using the new drug, but also the risks to patients if access to this new medicine is denied or delayed. Up to now, industry and senior FDA staff have not seized the opportunity to demonstrate at these public forums the utility of the concept of employing "reasonable risk" as an approval criterion. Rather, the public has been led to believe that drugs can and should be "absolutely safe," which industry believes is in practice impossible. While in principle the advisory board panel meeting process could provide such public education, industry and the FDA have not yet worked sufficiently hard to attain that goal.

\section{DISCUSSION AND DE NOVO “THINKING OUTSIDE THE BOX”}

The initial portion of the interviews we conducted involved interviewees responding to our written statements or questions on a scale of one to five, and indicating to us which of the issues raised were of sufficient importance or complexity that they wanted us to come back to them later on in the interview. Near the end of each interview with both industry and FDA respondents, we posed the following question orally: "If you had the opportunity to create the FDA de novo, on what would you focus your efforts, and five years from now, in what ways would you hope the FDA would differ most markedly from what it is today?" As a practical matter, in the final portions of our interviews respondents iterated back and forth on revisiting 
and expanding on early questions, and on opining on the de novo hypothetical. Our summary also mixes them.

Although respondents' comments ranged widely, three sets of issues were raised repeatedly, in some cases both by industry and the FDA. First, industry accepts the notion that the onus of developing new medicines is on them, and not on the FDA. They accept that the missions of the FDA are to ensure the safety and efficacy of new medicines, and to advance the public health by "helping to speed innovations that make medicines and foods more effective, safer and more affordable." The missions and tasks facing the FDA differ from those facing industry. A strength of the FDA to this point is that its mandate has been perceived by industry, the FDA and the public as being limited to evaluating medications based on scientific criteria involving safety and efficacy, and in particular, not on economic criteria involving comparative costs and benefits. Industry is concerned that with closer collaborations between the FDA and the Centers for Medicare and Medicaid Services, this valuable separation of mandates might become blurred, as it has already in member states of the EMEA. This blurring is likely to expand as the Medicare Part D prescription drug benefit is implemented in 2006.

Second, industry and much of the current FDA senior leadership believes that over the years, the FDA has focused disproportionate attention on the first of the two missions (product safety and efficacy), at the expense of meeting the second mission (helping speed innovation), although when we conducted our interviews (in Spring 2004, prior to the voluntary withdrawal of Vioxx), industry believed it was observing some welcome signs of change. With respect to "helping to speed innovations," guidance from and collaboration with the FDA can have significant positive impacts on how quickly and successfully pharmaceutical and biotech firms carry out their drug development efforts. While the onus of drug development is on industry, 
there is much room for closer collaboration between industry and the FDA that promotes the public health, particularly by sharing information during early development stages in which the FDA's valuable knowledge involving classes of compounds and molecule structures could help industry avoid failures and needlessly placing patients at risk.

Third, a persistent theme we heard from industry interviews (and to some extent, also from the FDA) was that communications processes with the FDA needed to be better managed. Increased communication and interactions with the FDA were viewed by industry as substantially increasing information transparency and reducing information asymmetry between the FDA and sponsors, thereby mitigating development uncertainty and risk, making the inherently risk drug development process more predictable. To be effective, however, these increased communications efforts must be coupled with standard performance metrics and best practices across the FDA's therapeutic divisions. Currently industry believes there is enormous variability across the FDA's 17 therapeutic divisions and areas in the quality of communications between the FDA and industry.

Both industry and FDA respondents recognized the existence of an inherent tension, however, between credibly regulating an industry and working with it to facilitate development of innovative products, i.e., between gate-keeping and development. FDA officials expressed particular strong caution that their supportive role be confined to collaboration, and not include extensive partnering. A delicate balance must be maintained between attempting to accommodate industry's desires to know and the agency's need to safeguard its decision-making process. That being said, both industry and the FDA recognize that later phase information communications are by necessity likely to be more constrained than those possible at earlier stages of the drug development process. The uniformly high valuation of additional Phase II 
informal communications voiced both by FDA and industry may signal that such efforts will be forthcoming. However, the discordant FDA-industry valuations of such additional communications during the earlier drug development phases are notable. Both industry and FDA officials stated that these issues merit careful consideration as the FDA carries out its "Critical Pathways" initiatives with industry, academia, other government agencies, and the public.

Finally, our "de novo" questions also yielded several less commonly voiced opinions. Among these were the following:

- Redefine the medical reviewer position at the FDA and mix it in with service at the NIH and CDC. Make the job description more attractive, and perhaps institute a fellowship program.

- Involve the medical associations more in the review process, particularly during the advisory board panel meeting.

- Have one toxicology unit serve all therapeutic divisions. More generally, the various therapeutic divisions are aligned too separately as silos, and instead there needs to be more cross-division consultation.

- Make the FDA more distant from the political process. The Commissioner of the FDA should be just as independent of the executive and legislative branches as is the Chairman of the Board of Governors of the Federal Reserve.

- Split regulatory supervision of food and agriculture off from the FDA, making it focused only on the safety and efficacy of medicines. Asking senior staff to be conversant with such wide-ranging issues involving food and drugs is practically unreasonable. 


\section{RECOMMENDATIONS: THEMES FROM QUALITATIVE DISCUSSIONS}

The less structured qualitative portions of our confidential interviews with 49 industry and eight senior FDA officials repeatedly focused on a number of suggested policy recommendations, which interviewees believed could lead to improved regulatory interactions between industry and the FDA and make the drug development and regulatory process more efficient, without compromising patient safety and welfare. Among those voiced most frequently and with the greatest intensity wee the following:

- Institute metrics and goals analogous to those present in the Prescription Drug User Fee Acts of 1992, 1997 and 2002 into the drug development portion of interactions with the agency. Sponsor companies would be charged reasonable levels of user fees for increased interactions during Phases II and III, not just upon submitting for review approval a New Drug Application or a Biologic License Application. The funds from sponsors would be used to hire additional, well qualified FDA staff to increase the interactions and information flows with sponsors.

- Contract with an independent consultant to identify divisions that are managed well, and that have been successful in fulfilling both portions of the FDA mission statement. Implement and regularly monitor the diffusion of standard best practices from the topperforming divisions across the entire set of agency divisions. Report on progress of the diffusion of best practices across therapeutic areas in the PDUFA annual report.

- Establish an oversight board consisting of industry, agency officials, and premier external scientists (possibly at NIH or CDC), that convenes at a minimum once a year to evaluate retrospectively the development and regulatory history of a randomly selected number of completed and terminated drug development projects - both successful and failed. This 
group would provide feedback to the FDA and industry and present actionable recommendations to improve the regulatory and development process.

- Establish an exchange program involving industry and FDA scientists that exposes industry to the FDA and vice-versa. To mitigate subsequent potential conflicts of interest, the FDA scientists would need to function in an industry project outside the review domain of the division employing them at the FDA.

- Collaborate on creating a more structured and effective dispute resolution system. The current ombudsman system is rarely, if ever used, and informal processes are inconsistent, creating unnecessary uncertainty and delaying decision-making.

- Establish a knowledge data base, using modern information technology, that stores and makes available information on issues concerning classes of compounds and molecule structures. Because the FDA is de facto the custodian of a knowledge base encompassing a much wider range of molecules and safety issues than is known by any in industry, it has a unique opportunity to provide developers with important information concerning safety and efficacy. These data could also be used in simulations and modeling involving safety and toxicity issues, projected both backwards and forwards. Certain proprietary and intellectual property issues will need to be addressed to facilitate the sharing of such information. However, allowing companies in ignorance to conduct clinical trials with compounds that share similar important properties with compounds that have previous safety or toxicity failures is inconsistent with protecting the public health.

Finally, we believe it important to bear in mind that issues involving interactions between the FDA and industry - the focus of this research - constitute only a portion of the much larger puzzle of why the recent substantially larger R\&D efforts by industry have not yielded 
commensurate growth in the number of NDAs/BLAs submitted for approval to the FDA. While resolution of the communication and management issues between the FDA and industry would improve substantially the drug development process and make it more efficient, these process solutions are not a substitute for the underlying discovery and development of innovative therapeutics. Ultimately, both industry and the FDA believe the onus of drug development rests with the pharmaceutical and biotechnology industries. The complementary missions of the FDA are to continue to ensure the safety and efficacy of medicines, and simultaneously to advance the public health by helping to speed innovations that make medicines accessible to the patients who need them.

\section{STUDY LIMITATIONS}

Our study has a number of limitations. Although the study team took considerable care in developing and testing the quantitative and qualitative questionnaires, in retrospect it is clear the questions could have been enhanced to delve deeper into some of the key issues that emerged. More pointed questions would have been extremely valuable in elucidating distinctions when the interviewee responded by giving a weighted average response. For example, interviewees often responded with the answer "fair" or "variable" to the questions regarding medical reviewers. Refinement of these questions to solicit feedback on a per division basis or with percentage estimates might have been more useful.

The sample of companies interviewed during this research was not chosen at random from a listing of biotech, pharmaceutical, and CRO companies. It was important for the purpose of the research to interview personnel at companies that had considerable experience with the FDA and drug development. The companies we chose to interview were admittedly nonrandom, and quite successful - as of April 2004, they had a total market capitalization of more 
than one trillion dollars. A random sampling of companies engaged in drug development would not have ensured coverage of companies with significant development experience. While not proven, we believe that the sample is representative of the major stakeholders of drug development within industry.

Virtually all the industry interviewees were engaged in R\&D or regulatory activities. General management and senior executives (e.g. chief executive officer, chief financial officer) were not interviewed. Their opinions on interactions with and evaluations of the FDA might be very different given the greater pressure they face from the investment community and shareholders.

Similarly, the eight senior FDA officials interviewed may not be representative of the entire leadership at the FDA. While the names of several of the FDA interviewees were provided by the study team, the majority of the eight FDA interviewees were selected by the FDA.

Finally, these interviews were conducted in Spring 2004, several months before Merck's voluntary withdrawal of Vioxx on September 30, 2004, and also before Congressional hearings on the safety of antidepressant medications for pediatric populations. The opinions and views of both industry and FDA interviewees may have changed over the intervening time period. 


\section{FOOTNOTES}

${ }^{3}$ U.S. Department of Health and Human Services, Food and Drug Administration, Innovation or Stagnation? Challenge and Opportunity on the Critical Path to New Medical Products, March 2004, Figures 1 and 2, p. 2.

${ }^{4}$ See Joseph A. DiMasi, Ronald W. Hansen and Henry G. Grabowski, "The Price of Innovation: New Estimates of Drug Development Costs", Journal of Health Economics, 22(2), 2003:151-186; and James Gilbert, P. Henske and

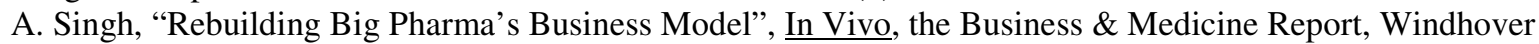
Information, 21(10), November 2003.

${ }^{5}$ Ernst R. Berndt, Adrian H. B. Gottschalk, Tomas J. Philipson and Matthew W. Strobeck, "Industry Funding of the FDA: Effects of PDUFA on Approval Times and Withdrawal Rates". In press, Nature Reviews: Drug Discovery, forthcoming, May 2005. This paper references numerous earlier studies as well.

${ }^{6}$ Computed as unweighted sample means. Data from Janice M. Reichert, "Trends in Development and Approval Times for New Therapeutics in the United States", Nature Reviews: Drug Discovery, 2, September 2003:695-702.

${ }^{7}$ Ernst R. Berndt, Adrian H. B. Gottschalk and Matthew W. Strobeck, "Critical Issues in Re-Engineering the Drug Development Process: Results from a Survey of Industry and the FDA", Cambridge, MA: MIT Sloan School of Management, draft manuscript, 4 August 2004, 63 pp.

${ }^{8}$ This figure is taken from data cited by M. P. Mathiew, ed., "Development Pipeline Attrition", PAREXEL Pharmaceutical R\&D Statistical Sourcebook 2003/2004, Waltham MA: 2003, p. 184, based on studies at the Tufts Center for the Study of Drug Development, Hambrecht \& Quist estimates.

${ }^{9}$ For additional discussion of the various components of preclinical and clinical development, see, for example, Joseph A. Dimasi, "New Drug Development in the United States from 1963 to 1999", Clinical and Pharmaceutical Therapeutics, 69, 2001:286-296; and Dimasi, "The Value of Improving the Productivity of the Drug Development Process: Faster Times and Better Decisions", Pharmacoeconomics, 20;Supp.3, 2002:1-10, and the references cited therein

${ }^{10}$ The probabilities of a compound moving from one phase to the next are approximate, and should not necessarily be interpreted as incorporating recent trends. It is also worth noting that probabilities of success differ for biologics and chemicals, and that the numbers quoted here are averages across all NMEs.

${ }^{11}$ Although we know of no firm data, in our survey a substantial number of interviewees expressed the view that very recent track record for Phase III transitions into NDAs/BLAs is less than 64\%.

${ }^{12}$ See DiMasi [2002].

${ }^{13}$ See the reference in footnote 5 above. That version now incorporates several corrections of the draft document provided us by interviewees.

${ }^{14}$ Unless discussed explicitly in the text, mean differences between pharmaceutical and biotechnology/biopharmaceutical company responses were not statistically significant. Average responses of the $\mathrm{CRO}$ respondents were also very similar.

${ }^{15}$ U. S. Food and Drug Administration [2004], p. 23. 